\title{
Effet du climat tropical humide sur la consommation d'aliment et d'eau et sur la vitesse de croissance de taurillons Créoles en Guadeloupe
}

\author{
P. BERBIGIER \\ I.N.R.A., Station de Bioclimatologie, \\ Station de Recherches zootechniques, \\ Centre de Recherches Antilles-Guyane, Domaine Duclos, F 97170 Petit-Bourg
}

\section{Résumé}

Nous avons étudié l'effet du climat tropical sur les quantités de matière sèche et d'eau ingérées, ainsi que sur la vitesse de croissance de taurillons Créoles en Guadeloupe (Antilles Françaises). Seize taurillons Créoles ont été affectés à quatre traitements, selon un modèle factoriel $2^{2}$ ( 2 situations climatiques "avec» et "sans abri », et 2 régimes, concentré ad libitum et fourrage ad libitum supplémenté avec du concentré).

Les animaux du régime "concentré » présentent, dans la situation «sans abri », une diminution de 8,5 p. 100 de la quantité de matière sèche ingérée par rapport aux animaux abrités. La différence est moindre $(2,8$ p. 100) pour les animaux du régime "fourrage». La vitesse de croissance n'est pas affectée par la situation climatique.

L'analyse en régression multiple de la quantité de matière sèche ingérée montre un faible effet de certains paramètres climatiques sur les animaux non abrités du régime "concentré », mais pas sur les animaux abrités. Pour tous les animaux du régime "fourrage », le principal facteur de variation est le taux de matière sèche de celui-ci.

L'analyse en régression multiple de la quantité d'eau totale ingérée montre un effet significatif du rayonnement global, même sur les animaux abrités : cependant, au-dessus de $1500-2000 \mathrm{~J} \cdot \mathrm{cm}^{-2}$ jour $^{-1}$, celle-ci augmente plus vite avec le rayonnement chez les animaux non abrités.

Les taurillons Créoles sont bien adaptés à leur environnement thermique. Cependant, ils doivent utiliser l'évaporation pour assurer leur thermorégulation et boire plus lorsqu'ils sont sans abri pour compenser les pertes évaporatives.

\section{Introduction}

Certains paramètres physiologiques caractéristiques de la contrainte climatique, en particulier le rythme respiratoire, augmentent en milieu chaud lorsque la thermolyse ne peut plus s'effectuer par la voie sensible. Cependant, si la thermorégulation par la voie latente (transpiration, évaporation respiratoire) est efficace, l'animal ne subit pas de «stress » thermique (BIANCA, 1968). Toutefois, il doit boire davantage pour compenser ses pertes en vapeur d'eau. 
Si la contrainte climatique augmente, il ingère moins de nourriture pour limiter sa production de chaleur, d'où une réduction des performances (KLEIBER, 1962 ; Bianca, 1976 ; SEIF, Johnson \& Lippincott, 1979).

Les travaux effectués jusqu'ici (Ingraham, Stanley \& Wagner, 1979 ; Johnson, 1980 ; ANSELL, 1981) concernent très souvent les vaches laitières issues de pays tempérés, très productives mais peu thermotolérantes. Nous avons cherché, dans le cadre d'une étude sur les bovins Créoles en Guadeloupe, à déterminer l'impact du climat tropical sur la consommation d'aliments et d'eau et les performances de ces animaux. Il s'agit donc d'un travaił réalisé dans un environnement climatique naturel, et sur des animaux de souches locales, qui avaient été très peu étudiés auparavant. En particulier, l'adaptation de ces animaux au climat tropical, souvent posée comme une évidence, mérite d'être vérifiée.

\section{Matéricl et méthodes}

L'expérience se déroule de septembre à mai dans la partie Nord de la Guadeloupe $\left(16^{\circ} \mathrm{N}\right)$, dans une zone dont le climat se caractérise par un rayonnement solaire intense, une humidité très élevée mais une pluviométrie réduite en saison sèche. Elle consiste à comparer, en fonction des différents paramètres du microclimat au niveau des animaux, la consommation d'aliment et d'eau, ainsi que la vitesse de croissance de taurillons Créoles à l'attache, placés soit dans des stalles non protégées des intempéries, soit dans une étable ouverte sur les côtés et munie d'un toit en tôle ondulée. Dans les deux cas la nourriture est protégée de la pluie par des contrevents faisant face à la direction de l'alizé. De plus, pour étudier le rôle de l'aliment ingéré, deux rations différentes sont distribuées à la moitié des animaux soumis à chacune des situations climatiques.

A l'issue d'une période préexpérimentale où les animaux sont placés à l'attache dans l'étable et sont nourris de manière identique, 16 taurillons d'un poids initial (moyenne \pm écart type) de $167 \pm 20 \mathrm{~kg}$ à un âge de $346 \pm 98$ jours sont répartis en quatre blocs en fonction du poids, du gain de poids moyen quotidien (GMQ) et de l'âge. Les animaux de chaque bloc sont ensuite soumis à l'un des 4 traitements suivant un schéma factoriel $2^{2}$ (deux situations climatiques et deux régimes alimentaires).

\section{A. Alimentation}

Deux lots de taurillons reçoivent un régime «concentré » composé d'aliment concentré (son de blé 68 p. 100 , maïs 17,5 p. 100 , mélasse 10 p. 100 , urée 1,5 p. 100) distribué ad libitum, et de fourrage vert de prairie naturelle («petit foin ») coupé chaque jour, à raison de 2 p. 100 du poids vif en matière fraîche. Les animaux ne consommant jamais la totalité du fourrage, celui-ci est considéré comme distribué ad libitum. Le fourrage représente respectivement 18 p. 100 et 22 p. 100 de la matière sèche (MS) ingérée pour les animaux avec et sans abri.

Les deux autres lots de taurillons reçoivent un régime «fourrage» composé du même fourrage distribué ad libitum et de concentré (de 2 à $2,5 \mathrm{~kg}$ d'aliment brut 
par animal et par jour); le concentré est toujours totalement consommé. Les animaux avec ou sans abri ingèrent 32 p. 100 de la MS sous forme de concentré. Pour des raisons pratiques, le complément n'est pas ajusté au poids des taurillons de manière très stricte : aussi a-t-il été introduit comme variable explicative dans l'analyse en régression multiple.

La teneur en matière sèche du fourrage est extrêmement variable selon la saison (20 à 70 p. 100), entraînant une grande variabilité des quantités de matière sèche ingérée en fourrage (de 40 à $150 \mathrm{~g}$ de matière sèche par $\mathrm{kg}{ }^{0.75}$ ). L'énergie métabolisable et la teneur en azote des composants de la ration (moyenne sur la période expérimentale) établies d'après les tables de valeur nutritive des aliments publiées par l'I.N.R.A. (I.N.R.A., 1978) et l'analyse du fourrage, sont respectivement $\mathrm{EM}=2,34 \mathrm{Mcal} / \mathrm{kg}$ de $\mathrm{MS}$ et $\mathrm{MAT}=180 \mathrm{~g} / \mathrm{kg}$ de $\mathrm{MS}$ pour le concentré, $\mathrm{EM}=1,42 \mathrm{Mcal} / \mathrm{kg}$ de $\mathrm{MS}$ et $\mathrm{MAT}=78 \mathrm{~g} / \mathrm{kg}$ de MS pour le fourrage.

L'eau est distribuée ad libitum au moyen d'abreuvoirs automatiques.

\section{B. Mesures de consommation et de croissance}

Les quantités de concentré et de fourrage brut ingérés sont mesurées chaque jour pour chaque animal, pendant 4 jours de la semaine. La quantité d'eau bue (une mesure globale par traitement) est mesurée en même temps. Les teneurs en matière sèche du concentré et du fourrage proposés et refusés sont déterminées chaque matin. Les animaux sont pesés tous les 28 jours. Les mesures effectuées pendant les 65 jours suivant le début des traitements ne sont pas prises en compte, la croissance des animaux n'étant pas stabilisée. Les observations retenues sont faites pendant une période de 28 semaines entre novembre et mai (101 jours de mesure). Au début de cette période, les poids moyens des taurillons sont de $236 \pm 16 \mathrm{~kg}$ (régime «concentré » sans abri), $230 \pm 23 \mathrm{~kg}$ (régime «concentré 》 avec abri), $215 \pm 32 \mathrm{~kg}$ (régime «fourrage » sans abri), et $214 \pm 27 \mathrm{~kg}$ (régime «fourrage» avec abri).

\section{Données climatiques}

Les températures et humidités relatives maximales et minimales à 2 mètres de hauteur (Tamax, Tamin, HRmax, HRmin), la pluviométrie et le rayonnement global incident sur une surface horizontale $(\mathrm{Rg})$ sont relevés quotidiennement dans un parc météorologique situé à proximité des animaux. La température ambiante maximale et l'humidité relative minimale permettent de calculer une température de point de rosée (Tr), plus représentative de la quantité de vapeur d'eau présente dans l'air, et qui est utilisée par la suite de préférence à HRmin. La vitesse du vent est relevée chaque jour à l'aide d'anémomètres à coupelles placés au niveau des animaux, à l'intérieur et à l'extérieur (Vin et Vex). Les relevés du pluviomètre et des anémomètres ont lieu à $7 \mathrm{~h}$ (heure locale). De plus, une mesure de la température du corps noir (boule de $10 \mathrm{~cm}$ de diamètre) qui intègre l'effet de la température ambiante, du rayonnement et du vent, est faite 2 fois par semaine au voisinage des animaux, dans les deux situations climatiques, aux environs de 12 heures. Quelques mesures nocturnes sont également effectuées. 


\section{Analyse statistique}

Deux types d'analyses sont effectuées :

- Une analyse de variance, sur les moyennes par animal, de la matière sèche (MS) ingérée, du gain de poids moyen quotidien (GMQ), de l'indice de consommation (IC), qui permet de tester la signification statistique des différences entre traitements. Le modèle utilisé est un modèle factoriel $2^{2}$, avec les animaux répartis en 4 blocs (SNEDECOR \& COCHRAN, 1971). De plus, les différences de consommation de concentré et de fourrage entre les animaux des régimes "concentré » à l'intérieur et à l'extérieur sont analysées à l'aide de tests « $t$ » (SNedecor \& Cochran, 1971). La consommation d'eau, mesurée globalement pour chaque traitement, ne peut faire l'objet d'une telle analyse.

- Une analyse en régression linéaire multiple progressive qui permet de tester l'effet de différentes variables explicatives sur les moyennes journalières de la consommation de matière sèche et d'eau pour chaque traitement. Les variables explicatives les moins significatives sont successivement éliminées, pour obtenir un modèle minimum (DAGNelie, 1975). Les variables explicatives choisies sont :

- Les données climatiques (Tamax, Tamin, V, Rg, pluie); l'humidité relative maximale, quasi-constante, n'est pas prise en compte.

- Les caractéristiques du fourrage (teneur en matières azotées totales MAT, teneur en matière sèche MS).

- Le poids des animaux.

- La matière sèche ingérée de la deuxième composante d'un régime (concentré ou fourrage) pour l'étude de la quantité de matière sèche ingérée de la composante dominante.

- La matière sèche ingérée totale pour l'étude de la quantité d'eau ingérée.

Le concentré et le fourrage du régime «concentré » étant en fait distribués ad libitum, deux modèles séparés sont proposés.

\section{Résultats}

\section{A. Variables climatiques}

L'expérience s'étend sur la saison sèche, précédée et suivie d'une période pluvieuse (fig. 1). L'humidité relative maximale à $2 \mathrm{~m}$ est quasi-constante, et égale à $90 \mathrm{p} .100$

Les corrélations entre les différentes variables climatiques sont faibles (les coefficients de corrélation ne sont en général pas significativement différents de 0 ), à l'exception toutefois de celles obtenues entre :

- la température ambiante maximale Tamax et le rayonnement global $\operatorname{Rg}(r=0,688)$;

- Ia température minimale de la nuit précédente Tamin et le point de rosée du jour $\operatorname{Tr}(r=0,553)$; 

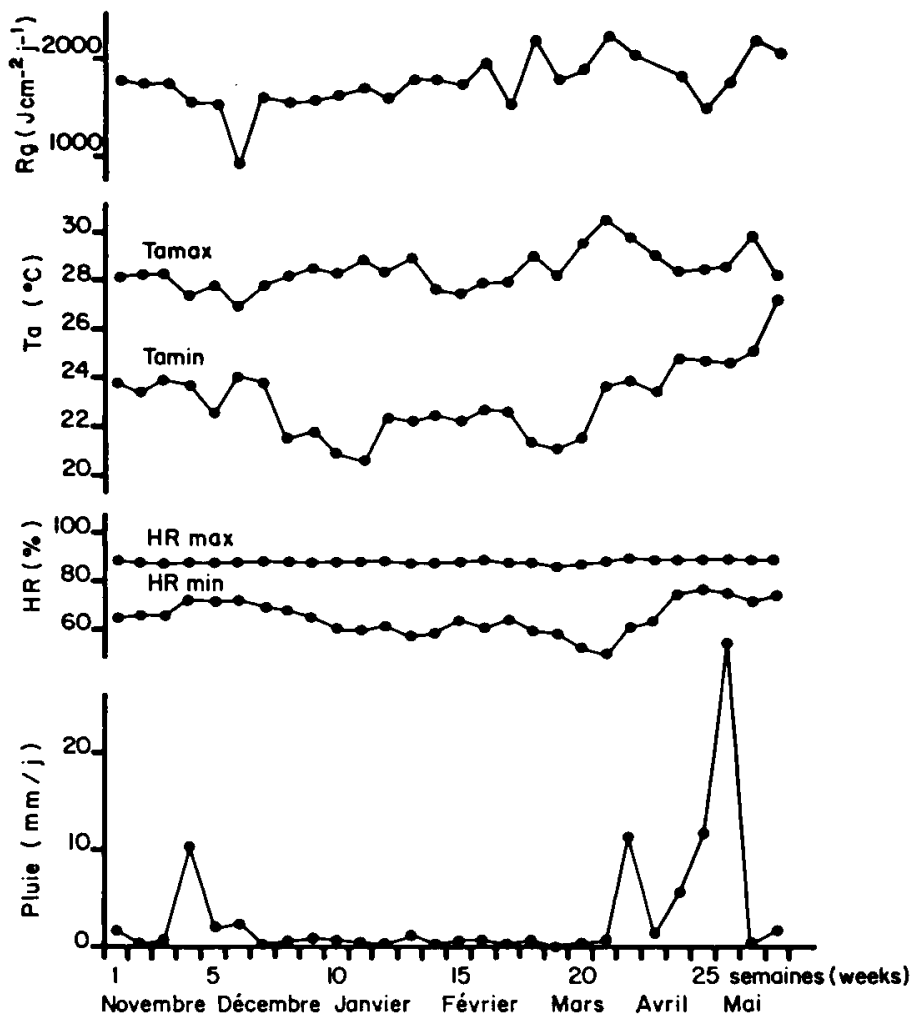

FiG. 1

Moyennes hebdomadaires des variables climatiques pendant la période expérimentale.

Weekly means of climatic variables during experimental period.

Rg est le rayonnement global, Tamax et Tamin sont les températures maximale et minimale, HRmax et $\mathrm{HRmin}$ les humidités relatives maximale et minimale.

$R g$ is overall radiation, Tamax and Tamin are maximum and minimum temperatures, HRmax and HRmin maximum and minimum relative humidities.

- le vent et la température minimale (respectivement $r=0,399$ et $r=0,430$ pour Vin et Vex);

- la température maximale et la pluviométrie PI $(r=0,235)$.

Au soleil, les températures du corps noir à 12 heures sont le plus souvent de l'ordre de 35 à $39^{\prime \prime} \mathrm{C}$, avec des maxima dépassant 42 " $\mathrm{C}$; sous l'abri protégeant les animaux, elles sont de l'ordre de $30{ }^{\circ} \mathrm{C}$. La nuit, leurs minima varient, dans les deux cas, entre 20 et $25^{\circ} \mathrm{C}$. 
B. Performances moyennes des taurillons pendant la période expérimentale

1. Consommation de matière sèche et vitesse de croissance (tabl. 1)

En moyenne, pendant la période expérimentale, les animaux ingèrent environ $6 \mathrm{~kg}$ de matière sèche par jour, quel que soit le régime. Le gain de poids vif est voisin de $0,75 \mathrm{~kg}$ par jour pour les régimes «concentré » et de $0,4 \mathrm{~kg}$ par jour pour les régimes «fourrage». L'interaction entre l'effet de la situation climatique et celui du type d'alimentation n'est significative pour aucune des variables étudiées.

\section{Tableau $1 \mathrm{a}$}

Valeurs moyennes ( \pm écart type) pendant la période expérimentale de la consommation de matière sèche,

du gain moyen quotidien et de l'indice de consommation.

Mean values ( \pm standard deviation) during the experimental period of dry matter intake, daily mean growth and feed conversion ratio.

\begin{tabular}{|c|c|c|c|c|}
\hline \multirow{2}{*}{$\begin{array}{c}\text { Situation climatique } \\
\text { Climatic situation } \\
\text { Alimentation } \\
\text { Diet } \\
\end{array}$} & \multicolumn{2}{|c|}{$\begin{array}{c}\text { Avec abri } \\
\text { With shelter }\end{array}$} & \multicolumn{2}{|c|}{$\begin{array}{c}\text { Sans abri } \\
\text { Without shelter }\end{array}$} \\
\hline & $\begin{array}{l}\text { «Concentré » } \\
\text { «Concentrate» }\end{array}$ & $\begin{array}{c}\text { «Fourrage» } \\
\text { «Forage» }\end{array}$ & $\begin{array}{l}\text { «Concentré » } \\
\text { «Concentrate» }\end{array}$ & $\begin{array}{c}\text { «Fourrage } \\
\text { «Forage }\end{array}$ \\
\hline $\begin{array}{l}\text { Matière sèche ingérée } \\
\text { en concentré }(\mathrm{kg} / \text { jour }) \\
\text { Concentrate dry matter } \\
\text { intake }(\mathrm{kg} / \text { day) }\end{array}$ & $4,52 \pm 0,24$ & $2,04 \pm 0$ & $5,46 \pm 0,71$ & $2,04 \pm 0$ \\
\hline $\begin{array}{l}\text { Matière sèche ingérée } \\
\text { en fourrage }(\mathrm{kg} / \text { jour }) \\
\text { Forage dry matter } \\
\text { intake }(\mathrm{kg} / \text { day })\end{array}$ & $1,31 \pm 0,04$ & $4,19 \pm 0,46$ & $1,18 \pm 0,10$ & $4,37 \pm 0,13$ \\
\hline $\begin{array}{l}\text { Matière sèche ingérée } \\
\text { totale }(\mathrm{kg} / \text { jour }) \\
\text { Total dry matter } \\
\text { intake }(\mathrm{kg} / \text { day })\end{array}$ & $5,83 \pm 0,28$ & $6,23 \pm 0,46$ & $6,37 \pm 0,33$ & $6,41 \pm 0,13$ \\
\hline $\begin{array}{l}\text { Gain moyen quotidien } \\
(\mathrm{kg} / \text { jour }) \ldots \ldots \ldots \ldots \\
\text { Daily mean growth } \\
(\mathrm{kg} / \text { day })\end{array}$ & $0,753 \pm 0,136$ & $0,372 \pm 0,041$ & $0,728 \pm 0,073$ & $0,444 \pm 0,059$ \\
\hline $\begin{array}{l}\text { Indice de consommation } \\
(\mathrm{kg} \mathrm{MSI} / \mathrm{kg} \mathrm{GMQ}) \\
\text { Feed conversion ratio } \\
(\mathrm{kg} \mathrm{DMI} / \mathrm{kg} \text { gain) }\end{array}$ & $7,93 \pm 1,34$ & $16,96 \pm 2,71$ & $8,78 \pm 0,59$ & $14,61 \pm 1,70$ \\
\hline
\end{tabular}




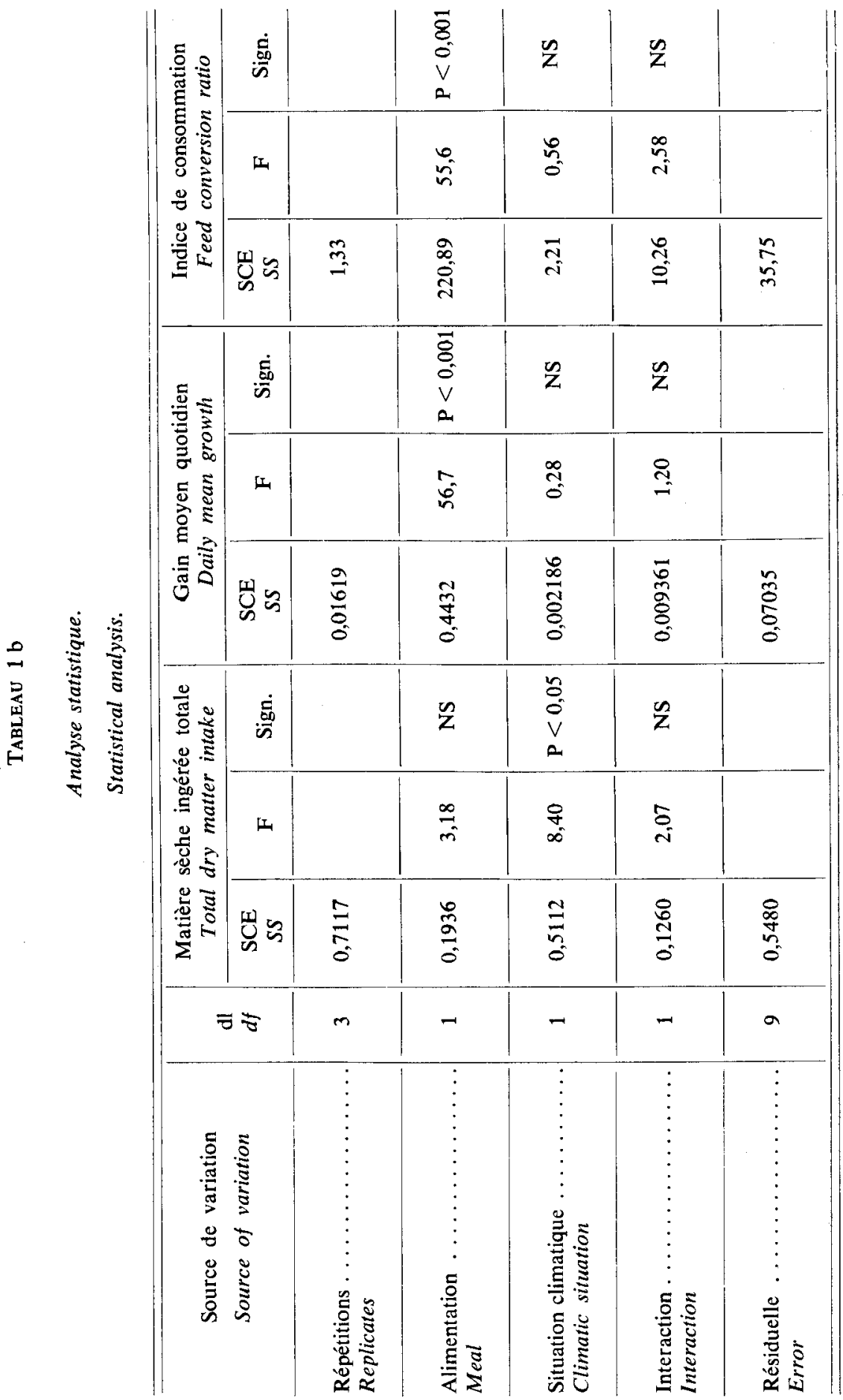


L'absence d'abri n'a pas d'effet sur le gain, ni sur l'indice de consommation. Par contre elle a un effet dépressif significatif $(p<0,05)$ sur la quantité de matière sèche totale ingérée, alors que le type d'alimentation n'en a pas. Il semble que cet effet dépressif soit plus fort dans le cas du régime "concentré »: cependant l'absence d'interaction significative, due peut-être au faible nombre d'animaux, ne permet pas de conclure en ce sens. S'ils sont privés d'abri, les taurillons soumis au régime «concentré » ingèrent davantage d'herbe $(1,31 \pm 0,04 \mathrm{~kg} / \mathrm{j}$ contre $1,18 \pm 0,10 \mathrm{~kg} / \mathrm{j}: \mathrm{p}=0,05)$ et moins de concentré $(4,52 \pm 0,24 \mathrm{~kg} / \mathrm{j}$ contre $5,46 \pm 0,71 \mathrm{~kg} / \mathrm{j}: \mathrm{p}<0,05)$.

\section{Consommation d'eau (tabl. 2)}

La consommation totale d'eau (aliment + boisson) avoisine $30 \mathrm{~kg}$ par jour et par animal, quel que soit le traitement, bien que les taurillons soumis au régime «concentré » boivent plus que ceux soumis au régime "fourrage», dont la teneur en MS est plus faible. Il apparaît que dans le cas du régime "concentré », la quantité d'eau ingérée, rapportée au $\mathrm{kg}$ de $\mathrm{MS}$ ingérée, par les animaux privés d'abri est d'environ 20 p. 100 supérieure à celle des animaux sous abri, alors que les valeurs sont sensiblement égales pour les animaux recevant le régime «fourrage *.

TABleau 2

Consommation d'eau (aliment et boisson), en valeur absolue et rapportée à la matière sèche ingérée.

Water intake (meal and drinking water) in absolute value and per $\mathrm{kg}$ dry matter intake.

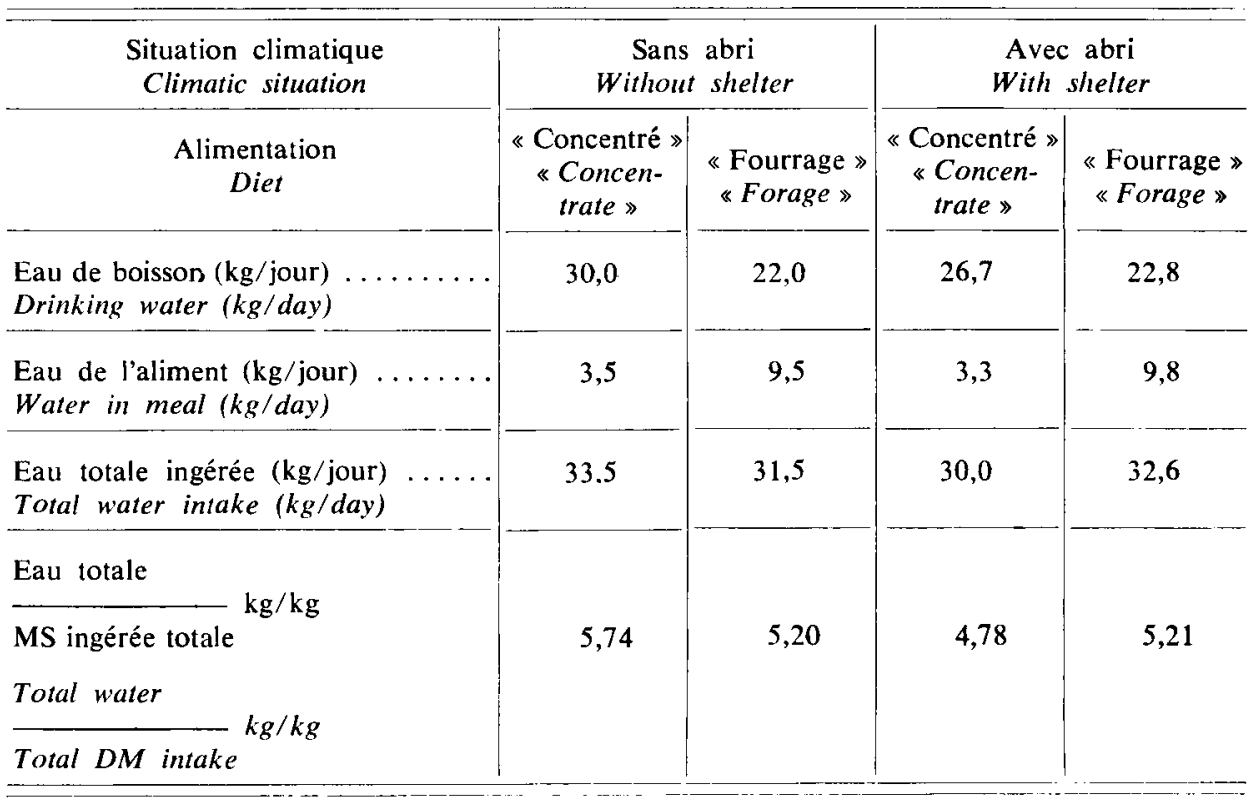




\section{Influence des facteurs climatiques sur la consommation de MS et d'eau}

\section{Quantité de matière sèche ingérée (tabl. 3)}

La teneur en matières azotées du fourrage n'a en aucun cas d'effet significatif et n'est pas portée sur le tableau 3.

\section{a) Régime «fourrage»}

La quantité de matière sèche de fourrage ingérée dépend principalement de sa teneur en matière sèche, quels que soient le régime et l'environnement climatique, comme le montrent également les équations de régression simple suivantes :

- Sans abri : MSI $=0,105 \mathrm{MS}+0,88\left(\mathrm{r}^{2}=0,67\right)$;

- Avec abri : MSI $=0,101$ MS $+1,15\left(\mathrm{r}^{2}=0,62\right)$

où MSI ( $\mathrm{kg}$ par animal et par jour) est la quantité de matière sèche de fourrage ingérée, et MS (p. 100) la teneur en matière sèche du fourrage. Le pouvoir explicatif de ces modèles est peu inférieur à celui des modèles minima de régression multiples présentés au tableau 3.

La quantité de matière sèche de fourrage ingérée dépend positivement de la quantité de matière sèche de concentré ingérée, mais est indépendante du poids vif des animaux.

Le rôle des variables du climat est plus fort lorsque les animaux sont privés d'abri que lorsqu'ils sont abrités. Dans le premier cas les effets sur la quantité de matière sèche de fourrage ingérée des variables suivantes sont significatifs $(p<0,05)$ : température maximale $(-0,190 \mathrm{~kg}$ par animal et par jour lorsque Tamax augmente de 1 ' $\mathrm{C}$ ), rayonnement global (+ $0,053 \mathrm{~kg}$ lorsque $\mathrm{Rg}$ augmente de $100 \mathrm{~J} \mathrm{~cm}^{-2}$ jour ${ }^{-1}$ ), et température de point de rosée $(+0,082 \mathrm{~kg}$ lorsque $\operatorname{Tr}$ augmente de 1 " $\mathrm{C})$. Dans le second cas, seul le rayonnement global $(+0,034 \mathrm{~kg}$ lorsque $\mathrm{Rg}$ augmente de $100 \mathrm{~J} \mathrm{~cm}^{-2}$ jour $\left.{ }^{-1}\right)$ atteint le seuil de signification $(\mathrm{p}<0,05)$.

\section{b) Régimes « concentré »}

La quantité de matière sèche de fourrage ingérée dépend à peu près des mêmes variables que celle des régimes «fourrage». Dans les deux situations climatiques, le taux de MS du fourrage est la variable explicative la plus importante $(+0,0316 \mathrm{~kg}$ par animal et par jour par p. 100 de MS dans la situation climatique «sans abri » et $+0,0276 \mathrm{~kg}$ « avec abri $», \mathrm{p}<0,01$ ). Contrairement aux régimes «fourrage », le poids vif des animaux a ici un effet positif. La température maximale (respectivement $-0,109$ et $-0,150 \mathrm{~kg}$ par $\left.{ }^{\circ} \mathrm{C}\right)$, le rayonnement global $(+0,0431$ et $0,0393 \mathrm{~kg}$ lorsque $\mathrm{Rg}$ augmente de $100 \mathrm{~J} \mathrm{~cm}^{-2} \times$ jour $\left.^{-1}\right)$, la température de point de rosée $(+0,051$ et $+0,052 \mathrm{~kg}$ par "C) agissent de façon significative $(\mathrm{p}<0,01)$ aussi bien sur les animaux "sans abri » que sur les animaux «avec abri». De plus le volume de précipitations joue un rôle positif sur la quantité de MS de fourrage ingérée par les animaux «sans abri » $\left(+5,72 \times 10^{-3} \mathrm{~kg}\right.$ par animal et par jour par millimètre de pluie, $\left.\mathrm{p}<0,05\right)$.

La quantité de matière sèche de concentré ingérée dépend négativement de la quantité de matière sèche de fourrage ingérée, positivement du poids vif des taurillons 


\section{TABLEAU 3}

Modèles minima de régression multiple expliquant la quantité de MS ingérée.

\begin{tabular}{|c|c|c|c|c|c|c|c|c|}
\hline & \multicolumn{3}{|c|}{$\begin{array}{c}\text { Matière sèche ingérée } \\
(\mathrm{kg} / \text { animal } \times \text { jour }) \\
\text { Dry matter intake } \\
(\mathrm{kg} / \text { animal } \times \text { day })\end{array}$} & \multirow{2}{*}{$\frac{\begin{array}{c}\text { Tamax } \\
\left({ }^{\circ} \mathrm{C}\right)\end{array}}{-}$} & \multirow{2}{*}{$\begin{array}{c}\begin{array}{c}\text { Tamin } \\
\left({ }^{\circ} \mathrm{C}\right)\end{array} \\
-\begin{array}{c}0,0675 \\
*\end{array}\end{array}$} & \multirow{2}{*}{$\frac{\mathrm{V}}{\left(\mathrm{ms}^{-1}\right)}$} & \multirow{2}{*}{$\begin{array}{c}\underset{\left(\mathrm{J} / \mathrm{cm}^{2} \times \mathrm{j}\right)}{-} \\
-\end{array}$} & \multirow{2}{*}{$\begin{array}{c}\begin{array}{c}\operatorname{Tr} \\
\left({ }^{\circ} \mathrm{C}\right)\end{array} \\
-\end{array}$} \\
\hline \multirow{4}{*}{ 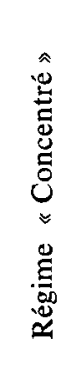 } & \multirow{4}{*}{ 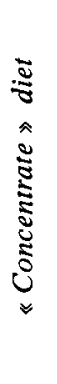 } & \multirow{2}{*}{ 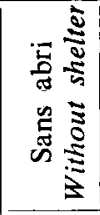 } & $\begin{array}{l}\text { Concentré } \\
\text { Concentrate }\end{array}$ & & & & & \\
\hline & & & $\begin{array}{l}\text { Fourrage } \\
\text { Forage }\end{array}$ & $-\underset{* *}{0,109}$ & - & - & $\underset{* *}{4,31 \times 10^{-4}}$ & $+\underset{* *}{0,0512}$ \\
\hline & & \multirow{2}{*}{ 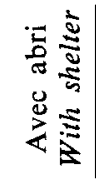 } & $\begin{array}{l}\text { Concentré } \\
\text { Concentrate }\end{array}$ & - & - & - & - & - \\
\hline & & & $\begin{array}{l}\text { Fourrage } \\
\text { Forage }\end{array}$ & $-\underset{* *}{0,150}$ & - & - & $\underset{* *}{3,93 \times 10^{-4}}$ & $+\underset{* *}{0,0516}$ \\
\hline \multirow{2}{*}{ 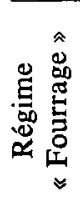 } & \multirow{2}{*}{ 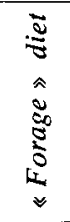 } & 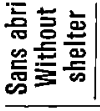 & $\begin{array}{l}\text { Fourrage } \\
\text { Forage }\end{array}$ & $\begin{array}{c}-0,190 \\
*\end{array}$ & 一 & - & $+\underset{*}{5,25} \times 10^{-4}$ & $\begin{array}{c}+0,0823 \\
*\end{array}$ \\
\hline & & $\mid$ & $\begin{array}{l}\text { Fourrage } \\
\text { Forage }\end{array}$ & - & - & - & $+\underset{*}{3,42 \times 10^{-4}}$ & 一 \\
\hline
\end{tabular}

Les valeurs numériques sont les coefficients de régression relatifs aux variables explicatives (*: $\mathrm{p}<0,05 ; * *: \mathrm{p}<0,01$ ). Tamax et Tamin sont les températures maximale et minimale, $\mathrm{V}$ la vitesse du vent, $\mathrm{Rg}$ le rayonnement global, $\mathrm{Tr}$ le point de rosée, $M S$ le taux de matière sèche du fourrage, MSI la quantité de matière sèche ingérée de l'autre composant de la ration, $\mathrm{R}^{2}$ le carré du coefficient de corrélation multiple.

et du taux de MS du fourrage (respectivement $+0,0186$ et $+0,0201 \mathrm{~kg}$ par animal et par jour par p. 100 de MS pour les situations «sans» et « avec abri »; p $<0,01$ dans les deux cas).

Le rôle des variables climatiques est faible. Cependant la température minimale de la nuit précédente $\left(-0,0675 \mathrm{~kg}\right.$ lorsque celle-ci augmente de $\left.1{ }^{\circ} \mathrm{C}, \mathrm{p}<0,05\right)$, le vent $\left(+0,283 \mathrm{~kg}\right.$ pour une augmentation de $\left.1 \mathrm{~ms}^{-1}, \mathrm{p}<0,05\right)$, la pluie $(+0,0119 \mathrm{~kg}$ par mm de pluie, $\mathrm{p}<0,05)$ ont une influence sur les animaux du régime "concentré » privés d'abri. Le contraste est net avec situation « avec abri » où il n'existe aucun effet significatif des variables climatiques.

\section{Quantité d'eau totale ingérée (aliment et boisson)}

Le tableau 4 donne les modèles minima de régression multiple obtenus. Seules les variables explicatives atteignant au moins une fois la signification y ont été prises 
Minimal multiple regression models explaining dry matter intake.

\begin{tabular}{|c|c|c|c|c|c|}
\hline $\begin{array}{c}\text { Pluie } \\
\text { (mm/jour) } \\
\text { Rain } \\
(\mathrm{mm} / \text { day })\end{array}$ & $\begin{array}{l}\text { MS } \\
(\%)\end{array}$ & $\begin{array}{c}\text { Poids }(\mathrm{kg}) \\
\text { Weight }(\mathrm{kg})\end{array}$ & $\begin{array}{c}\text { MSI } \\
(\mathrm{kg} / \mathrm{an} \times \text { jour }) \\
(\mathrm{kg} / \mathrm{an} \times \text { day })\end{array}$ & $\begin{array}{l}\text { Constante } \\
\text { Constant }\end{array}$ & $\mathrm{R}^{2}$ \\
\hline$+\underset{*}{0,0119}$ & $+\underset{* *}{0,0186}$ & $+\underset{* *}{0,00735}$ & $\begin{array}{c}-0,374 \\
*\end{array}$ & $+3,43$ & 0,35 \\
\hline$+\underset{*}{0,00572}$ & $+\underset{* * k}{0,0316}$ & $+\underset{* *}{0,00360}$ & - & $+0,44$ & 0,67 \\
\hline - & $+\underset{* *}{0,0201}$ & $+\underset{* *}{0,00802}$ & $\begin{array}{c}-0,454 \\
* *\end{array}$ & $+2,64$ & 0,23 \\
\hline- & $+\underset{* *}{0,0276}$ & $+\underset{* *}{0,00503}$ & 一 & $+1,31$ & 0,55 \\
\hline - & $+\underset{* *}{0,120}$ & - & $+\underset{* * *}{2,11}$ & $-1,15$ & 0,81 \\
\hline - & $+\underset{* *}{0,112}$ & - & $+\underset{* *}{2,46}$ & $-4,80$ & 0,77 \\
\hline
\end{tabular}

The numerical values are regression coefficients relative to explicative variables $(* ; p<0.05 ;$ $*: p<0.01$ ). Tamax and Tamin are maximal and minimal temperatures, $V$ is wind velocity, $\mathrm{Rg}$ overall radiation, $\mathrm{Tr}$ dew point, $\mathrm{MS}$ dry matter content of forage, MSI dry matter intake of the other component of the diet, $R^{2}$ square of multiple correlation coefficient.

en compte. En particulier, ni la quantité de MS totale ingérée ni la teneur en MS et en MAT du fourrage n'ont d'effet sur la quantité d'eau totale ingérée, malgré les variations très importantes de la teneur en MS (donc en eau) du fourrage.

Pour tous les régimes, la consommation d'eau augmente avec le poids de l'animal.

Parmi les variables climatiques, seul Ie rayonnement global, et, dans une moindre mesure la température de point de rosée et le vent, interviennent. Pour le régime «concentré », l'effet du rayonnement global est beaucoup plus important dans la situation «sans abri » que dans celle «avec abri», avec, semble-t-il, un seuil de divergence situé entre 1500 et $2000 \mathrm{~J} \mathrm{~cm}^{-2} \mathrm{j}^{-1}$ (fig. 2). Par contre, pour le régime "fourrage», le rayonnement a la même influence dans les deux situations.

La température du point de rosée intervient positivement sur l'ingestion d'eau dans le cas du traitement «fourrage sans abri ». Quant au vent, son rôle est faible. 


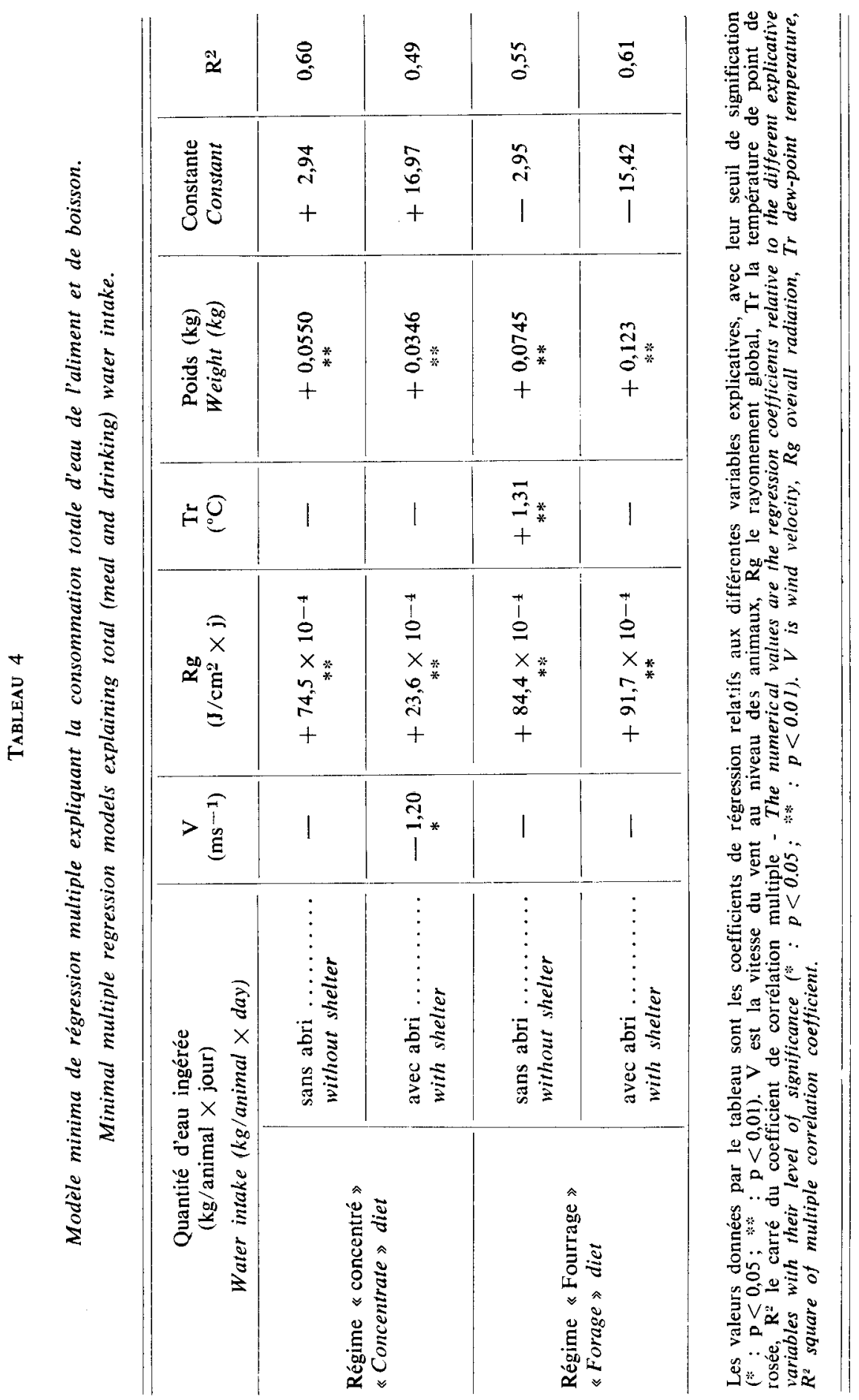




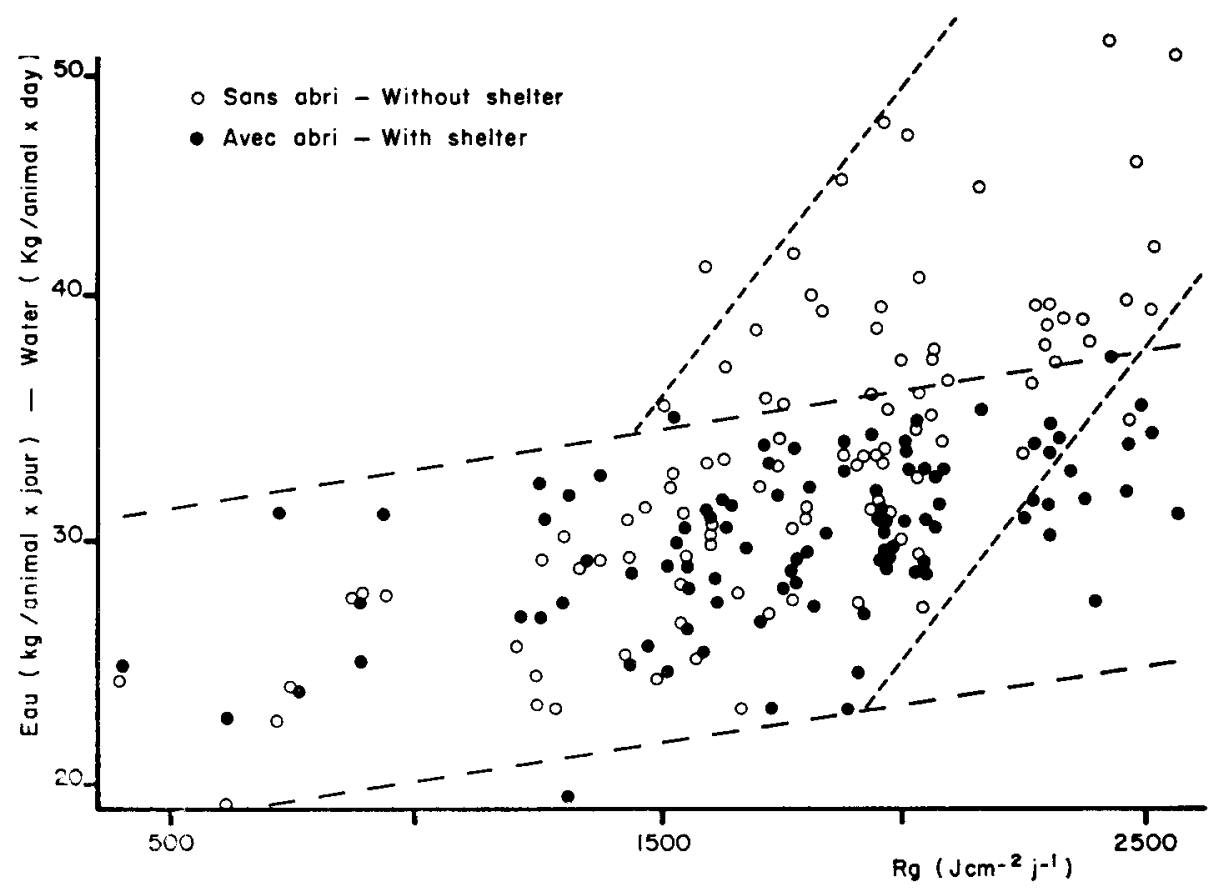

FIG. 2

Elfet du rayonnement global $\mathrm{Rg}$ sur la quantité d'eau totale ingérée (aliment et boisson) du régime «concentré » avec et sans abri.

Effect of overall radiation $\mathrm{Rg}$ on total (meal and drinking) water intake of the "concentrate» diet with and without shelter.

Les pointillés représentent les limites supérieures et inférieures des points représentatifs. Dotted lines represent upper and lower limits of representative spots.

\section{Discussion}

\section{A. Quantité de MS ingérée et gain de poids vif}

Le fait majeur ressortant de cette étude est le rôle réduit des variables climatiques, dans nos conditions expérimentales, sur la quantité de MS ingérée et le gain de poids vif des taurillons Créoles. La diminution faible mais significative des quantités de MS ingérée par les animaux privés d'abri montre que ces derniers subissent, du fait de la contrainte climatique, une réduction de leur appétit. De plus, dans ce cas, les animaux du régime "concentré » mangent plus d'herbe et moins de concentré que ceux qui sont sous l'abri : peut-être est-ce pour eux un moyen d'augmenter leur consommation d'eau en utilisant celle du fourrage. Curieusement ces différences entre situations climatiques n'ont aucun effet sur le gain de poids vif : la vitesse de croissance de nos taurillons Créoles n'est pas améliorée par l'ombrage. Peut-être ceci est-il 
dû à l'état sanitaire des animaux placés sous abri : nous avons alors noté plusieurs cas de diarrhée et un cas de météorisation, affections qui n’ont jamais été observées en l'absence d'abri.

L'analyse en régression multip'e de la quantité de MS ingérée journalière montre que, aussi bien pour le régime "concentré » que pour le régime "fourrage » limpact du climat, estimé à travers les variables qui le caractérisent, est plus fort dans la situation «sans abri » que dans la situation «avec abri». Ceci confirme donc que les animaux privés d'abri doivent moduler leur prise de nourriture en fonction de la contrainte climatique. Toutefois, dans le cas du fourrage du régime «concentré », le rôle des variables climatiques est du même ordre avec et sans abri. Ce phénomène, difficile à expliquer, porte cependant sur des quantités ingérées faibles (tabl. 1).

La quantité de matière sèche ingérée, selon qu'elle est composée de concentré ou de fourrage, ne dépend pas des mêmes variables climatiques. Dans le premier cas, pour les animaux privés d'abri, la consommation augmente lorsque la température minimale diminue (nuits fraîches), et lorsque le vent et la pluie, qui améliorent la thermolyse des animaux, augmentent. Le rayonnement solaire, qui est un facteur de contrainte thermique très important, n'apparaît pas dans le modèle minimum de régression multiple, mais ceci est peut-être lié au faible degré de signification de l'ensemble des effets climatiques. Dans le deuxième cas (fourrage des régimes «fourrage» et «concentré ») les variables climatiques ayant un effet sur la MS de fourrage ingérée sont différentes. Le comportement alimentaire des taurillons pourrait en être la cause, les animaux ingérant volontairement concentré et fourrage à des heures différentes. Cependant ceci reste du domaine de lhypothèse, seule une étude de leur comportement alimentaire et mérycique pouvant permettre de conclure. Le climat semble agir en deux sens : une action négative de la température maximale et une action positive du rayonnement global et, secondairement, du point de rosée. Le rôle négatif de la température maximale peut s'expliquer par une diminution de la consommation lors des journées les plus chaudes; dans le cas du rayonnement global et du point de rosée l'animal réagirait à la contrainte thermique en mangeant plus de fourrage frais pour augmenter sa consommation d'eau. Ces dernières variables ont en effet une action tout à fait similaire sur !es quantités de fourrage et d'eau totale ingérées dans le cas des régimes «fourrage». II faut cependant noter que la température maximale et le rayonnement global présentent une forte corrélation, ce qui rend toute conclusion aléatoire sur leurs rôles opposés.

De plus, il convient de rappeler que les effets climatiques directs, dans les régimes «fourrage», sont secondaires par rapport au rôle de la teneur en matière sèche du fourrage, qui peut être considérée comme un effet climatique indirect lié à la pluviométrie de la période précédant la mesure.

\section{B. Quantité d'eau ingérée}

La quantité d'eau ingérée (aliment + boisson) en moyenne pendant la période expérimentale ne scmble pas, en valeur absolue, très différente d'un traitement à l'autre malgré les différences de teneur en MS des aliments et l'exposition au soleil d'une partie des animaux. Il est à signaler que, pour des raisons techniques, la mesure de consommation d'eau du traitement "fourrage avec abri " est entachée d'une forte incertitude : les conclusions relatives à celui-ci doivent donc être tirées sous toutes réserves. 
L'analyse en régression multiple des quantités ingérées quotidiennes (aliment + boisson) montre que ni la quantité de MS ingérée ni la teneur en MS du fourrage n'agissent de façon significative sur la quantité d'eau totale ingérée. Ceci peut sembler surprenant, car, en pays tempéré, cette dernière est liée à la MS ingérée (I.N.R.A., 1978). Peut-être sagit-il d'une adaptation à la saison sèche où les animaux Créoles doivent assurer leur digestion malgré un fourrage très sec et des possibilités d'abreuvement réduites.

Par contre, l'effet des facteurs climatiques, principalement du rayonnement global, est primordial, même sous abri, où il intervient probablement en échauffant le toit en tôle, d'où une augmentation du rayonnement infra-rouge de celui-ci.

L'effet de l'abri sur le régime «fourrage» n'apparaît pas clairement : peut-être est-ce dû aux incertitudes de mesure relatives à ce traitement. En revanche, les animaux du régime «concentré » privés d'abri ingèrent plus d'eau que ceux placés sous abri dès que le rayonnement global dépasse le seuil mentionné plus haut ( $\$$ III D), probablement pour compenser les pertes d'eau par évaporation. Or le niveau de ce seuil est relativement bas, puisquiil est inférieur à la valeur moyenne journalière de $\mathrm{Rg}$ observée sur 3 ans $\left(1900 \mathrm{~J} \cdot \mathrm{cm}^{-2} \mathrm{j}^{-1}\right)$.

Les autres paramètres du climat ont une action faible ou nulle sur la consommation d'eau. Cependant l'effet du point de rosée, qui est toujours à la limite de la signification et atteint le niveau $\mathrm{p}<0,01$ en un cas, est à noter, car linfluence de $\mathrm{Rg}$ et $\mathrm{Tr}$ sur les quantités d'eau ingérées expliquent sans doute, comme nous l'avons vu plus haut, leur action sur la consommation de fourrage.

La réduction de l'ingestion volontaire d'aliment et de la croissance ainsi que l'augmentation de la quantité d'eau absorbée chez les bovins placés en ambiance chaude, sont des phénomènes classiques (THOMPSON, 1973). Cependant on ne trouve que très peu de travaux relatifs aux races bovines tropicales en milieu thermique non contrôlé. En chambre climatique, sur des génisses zébu de $300 \mathrm{~kg}$, SEIF, Johnson \& LippincotT (1979) observent une diminution de 19 p. 100 de l'ingestion volontaire d'aliment et une augmentation de 58 p. 100 de la quantité d'eau bue à $31^{\circ} \mathrm{C}$ par rapport à une température de référence de $10{ }^{\circ} \mathrm{C}$. Cependant, ni la composition de la ration ni sa teneur en eau n'étant précisées, une comparaison avec nos résultats est difficile.

Quant à l'effet des différents paramètres décrivant le climat tropical sur l'ingestion d'aliment et d'eau, il n’a, à notre connaissance, jamais été étudié.

\section{Conclusion}

Il semble que, dans nos conditions expérimentales (alimentation et boisson ad libitum) et dans les conditions climatiques de la partie Nord de la Guadeloupe, la consommation d'aliment et la croissance des taurillons Créoles soient peu affectées par la contrainte climatique. L'équilibre thermique est sans doute obtenu grâce à la thermolyse latente (évaporation), comme le montrent les quantités d'eau consommées et l'effet du climat sur celle-ci.

Malgré leur bonne adaptation, les taurillons sont donc obligés de mettre en jeu des mécanismes actifs de thermolyse dans la journée (transpiration et/ou évaporation respiratoire).

Accepté pour publication en janvier 1983. 


\section{Summary \\ Effect of humid tropical climate on food and water intake and weight gain of growing Creole bulls in Guadeloupe}

Dry matter and water intake as well as weight gain of Creole growing bulls were studied, as a function of climatic conditions in Guadeloupe (French West Indies). Sixteen Creole growing bulls were affected to four treatments following a $2^{2}$ factorial design ( 2 climatic situations, with and without shelter, and 2 diets : concentrate ad libitum and forage ad libitum supplemented with concentrate).

There was a $8.5 \mathrm{p} .100$ decrease in dry matter intake of the non-sheltered animals fed the concentrate diet as compared with the sheltered ones. The difference was less marked for animals fed forage $(2.8 \mathrm{p}$. 100). Weight gain was not affected by the climatic situation (tabl. 1).

Multiple regression analysis of dry matter intake showed a small effect of some climatic parameters on the non-sheltered animals fed the concentrate diet and no climatic effect on the sheltered ones. In forage-fed animals, the main factor was the dry matter content of the forage (tabl. 3).

Multiple regression analysis of total water intake showed a relevant effect of the overall radiation, even in sheltered animals : however beyond $1500-2000 \mathrm{~J} \cdot \mathrm{cm}^{-2}$, day ${ }^{-1}$, water intake of the non-sheltered animals increased very quickly with solar radiation, as compared to the sheltered ones (tabl. 4 and fig. 2).

The Creole growing bulls are well adapted to their thermal environment. Nevertheless, they must use evaporation to ensure thermoregulation and drink more when non-shaded to compensate for evaporation losses.

\section{Références bibliographiques}

ANSELL R.H., 1981. Extreme heat stress in dairy cattle and its allevation : a case report, 285-306. In : CLARK J.A., Environmental aspects of housing for animal production, Butterworths, London.

Bianca W., 1968. Thermoregulation, 97-118. In : Hafez E.S.E., Adaptation of domestic animals, Lea and Febiger, Philadelphia.

Bianca W., 1976. The significance of meteorology in animal production. Int. J. Biometeorol., 20, 139-156.

Ingraham R.H., Stanley R.W., Wagner W.C., 1979. Seasonal effects of tropical climate on shaded and nonshaded cows as measured by rectal temperature, adrenal cortex hormones, thyroid hormone, and milk production. Am. J. Vet. Res., 40, 1792-1797.

I.N.R.A., 1978. Alimentation des ruminants. 621 p., I.N.R.A. éd., route de Saint-Cyr, Versailles.

Dagnelie P., 1975. Théorie et méthodes statistiques. Tome 2, 463 p., Les Presses Agronomiques, Gembloux.

JoHnson H.D., 1980. Environmental management of cattle to minimize the stress of climatic change. Biometeorology, 7, part $2: 65-78$ (supplement to Int. J. Biometeor., 24).

KLeiber M., 1962. The fire of life. 454 p., John Wiley and sons, New York, London.

Seif S.M., Johnson H.D., LippincotT A.C., 1979. The effects of heat exposure $\left(31^{\circ} \mathrm{C}\right)$ on Zebu and Scottish Highland cattle. Int. J. Biometeor., 23, 9-14.

SNedecor G.W., Cochran W., 1971. Méthodes statistiques. 649 p., Association de Coordination Technique Agricole, Paris.

Thompson G.E., 1973. Review of the progress of dairy science. Climatic physiology of cattle. J. Dairy Res., 40, 441-473. 